\title{
Pain Coping Skills Training for Patients with Elevated Pain Catastrophizing who are Scheduled for Knee Arthroplasty: A Quasi-Experimental Study
}

\author{
Daniel L. Riddle, PT, PhD, Francis J. Keefe, PhD, William T. Nay, PhD, Daphne McKee, PhD, \\ David E. Attarian, M.D., F.A.C.S, and Mark P. Jensen, PhD \\ Departments of Physical Therapy and Orthopaedic Surgery (Riddle) and Psychiatry (Nay)Virginia \\ Commonwealth University, Richmond, VA; and Departments of Psychiatry and Behavioral \\ Sciences, Anesthesiology, Medicine and Psychology (Keefe) and Psychiatry and Behavioral \\ Sciences (McKee), and Orthopaedic Surgery (Attarian) Duke University, Durham, NC; and The \\ Department of Rehabilitation Medicine, University of Washington, Seattle, WA (Jensen)
}

\section{Abstract}

Objectives-To (1) describe a behavioral intervention designed for patients with elevated pain catastrophizing who are scheduled for knee arthroplasty, and (2) use a quasi-experimental design to evaluate the potential efficacy of the intervention on pain severity, catastrophizing cognitions, and disability.

Design-Quasi-experimental non-equivalent control group design with a 2 month follow-up.

Setting-Two university-based Orthopedic Surgery departments.

Participants-Adults scheduled for knee replacement surgery who reported elevated levels of pain catastrophizing. Patients were recruited from two clinics and were assessed prior to surgery and 2 months following surgery.

Intervention-A group of 18 patients received a psychologist directed pain coping skills training intervention comprising 8 sessions and the other group, a historical cohort of 45 patients, received usual care.

Main Outcome Measures-WOMAC Pain and Disability scores as well as scores on the Pain Catastrophizing Scale.

Results-Two months following surgery, the patients who received pain coping skills training reported significantly greater reductions in pain severity and catastrophizing, and greater improvements in function as compared to the usual care cohort.

Conclusion-Pain catastrophizing is known to increase risk of poor outcome following knee arthroplasty. The findings provide preliminary evidence that the treatment may be highly efficacious for reducing pain, catastrophizing, and disability, in patients reporting elevated

Corresponding author: Daniel L. Riddle, PT, PhD, Otto D. Payton Professor, Departments of Physical Therapy and Orthopaedic Surgery, PO Box 980224, 1200 East Broad Street, Room B-100, Virginia Commonwealth University, Richmond, Virginia 23298-0224, (p) 804-828-0234, (f) 804-828-8111, dlriddle@vcu.edu.

Material was presented in poster format at the Osteoarthritis Research Society International meeting in Brussels Belgium in September, 2010.

Publisher's Disclaimer: This is a PDF file of an unedited manuscript that has been accepted for publication. As a service to our customers we are providing this early version of the manuscript. The manuscript will undergo copyediting, typesetting, and review of the resulting proof before it is published in its final citable form. Please note that during the production process errors may be discovered which could affect the content, and all legal disclaimers that apply to the journal pertain. 
catastrophizing prior to knee arthroplasty. A randomized clinical trial is warranted to confirm these effects.

\section{Keywords}

knee; arthroplasty; distress; pain; arthritis; catastrophizing

Patients with severe knee arthritis typically report that pain is their most bothersome symptom. ${ }^{1 ; 2}$ Knee arthroplasty is generally an effective surgical procedure for patients with painful knee arthritis who have not responded to conservative treatment. ${ }^{1}$ These large group level effects on pain intensity indicate that knee replacement surgery is highly effective at reducing pain for many. However, there are a substantial number of patients who experience little pain improvement or, in some cases, worse pain following knee arthroplasty. For example, Brander and colleagues reported that $19 \%$ of patients in their cohort of 116 patients reported moderate to severe pain 6 months following surgery ${ }^{3}$ while Hawker and colleagues reported similar estimates 2 to 7 years following arthroplasty. ${ }^{4}$ Murray and colleagues found that $30 \%$ of their large cohort of 1429 patients reported moderate or severe pain 1 year after knee arthroplasty. 5

A substantial body of research has since been devoted to identifying predictors of persistent pain or poor function. ${ }^{6}$ Among the most consistent and powerful are psychological predictors, and a burgeoning body of literature suggests that pain catastrophizing is among the most important, if not the most important, psychological predictor of poor outcome. ${ }^{7-11}$ Although the specific mechanisms by which catastrophizing might influence pain have not yet been determined, a number of such mechanisms have been suggested. ${ }^{12}$ For example, it is possible that catastrophizing could have a direct influence on the neurophysiological mechanisms involved in pain processing. ${ }^{13}$ Finally, it has been proposed that catastrophizing may also lead to social responses (e.g. increases in attention from a concerned spouse) that may heighten patients' focus on and rumination about pain. ${ }^{14}$

The two purposes of this study were to: (1) describe a pain coping skills training intervention that our team developed specifically for a subgroup of patients scheduled for knee arthroplasty who also had high levels of pain catastrophizing, and (2) conduct a quasiexperimental study to evaluate the efficacy of this intervention on a consecutively recruited sample of patients, comparing the outcomes to a usual care cohort of patients with similarly high levels of pain catastrophizing but who did not receive pain coping skills training. Quasi-experimental studies are appropriate for use when innovative interventions are developed and compared to a reasonable control condition to obtain an initial estimate of efficacy and to determine if a randomized clinical trial is warranted. ${ }^{15}$ Because our study was preliminary in nature, we chose not to declare a single primary outcome variable but rather to examine effects on three important outcomes: self-reported pain severity, function and pain catastrophizing. To our knowledge, this is the first study to test the effects of a pain coping intervention in patients with knee arthroplasty.

\section{METHODS}

\section{Design}

This quasi-experimental (i.e., non-randomized) study compared treatment outcome between two independent cohorts, one that received the pain coping skills training and one that received usual care. 


\section{Participants}

Pain coping skills training cohort-Participants in the pain coping skills group were consecutively recruited between April and September, 2009 from two sites: (1) Virginia Commonwealth University Medical Center, Richmond, Virginia or (2) Duke University Medical Center, Durham, North Carolina. The patients had already consented to have knee arthroplasty and were consented to participate in the current study two to six weeks prior to their scheduled surgery. Patients were eligible to participate if they: (1) were scheduled for primary (not revision) unilateral knee arthroplasty and (2) scored a 16 or higher on the Pain Catastrophizing Scale (PCS). We found in a previous study that patients who scored a 16 or higher on the PCS had an increased risk of poor outcome following surgery. ${ }^{9}$ Patients were excluded if they could not speak or read English or if they did not sign a consent form. To identify patients who were eligible, we asked patients who met the other inclusionary criteria to complete the PCS. We screened a total of 56 patients for the study, of which 24 scored a 16 or higher on the PCS. Of the 24 patients who screened positive, a total of 18 (75\%) consented to participate in the study.

Usual care cohort-Participants in the usual care cohort study also consented to participate prior to surgery. These patients participated in a prospective cohort study conducted between December, 2005 and April, 2008 at Virginia Commonwealth University Medical Center, Richmond, Virginia. The study was designed to determine the association between various psychological measures and outcome following knee arthroplasty. ${ }^{9}$

Patients were recruited if they were scheduled for primary (not revision) unilateral knee arthroplasty. Patients were excluded if they could not speak or read English or if they did not sign a consent form. A total of 157 patients were recruited to complete both the baseline data and 2 month follow-up forms and $139(89 \%)$ actually completed follow-up data. A total of 45 of these 139 patients (32.3\%) scored a 16 or higher on the PCS and thus qualified for the current study. The demographic and knee symptom characteristics of the patients in the pain coping skills cohort and the usual care cohort are summarized in Table 1.

\section{Measures}

Participants in both cohorts completed three key questionnaires in addition to the demographic data summarized in Table 1.

Pain Catastrophizing Scale-The Pain Catastrophizing Scale (PCS) is a 13-item scale with scores ranging from 0 (no catastrophizing) to 52 (severe catastrophizing). The PCS was designed to capture the extent of a patient's negative or exaggerated orientation to pain and it addresses primary constructs of rumination, magnification and helplessness. ${ }^{16}$ Psychometric properties of the PCS have been studied extensively in a variety of patient populations having painful disorders, and found to be good to excellent. ${ }^{16-18}$ In addition, the PCS has been shown to be sensitive to psychosocial interventions designed to increase the use of adaptive coping strategies and decrease the use of maladaptive coping strategies, such as pain catastrophizing. ${ }^{19-22}$

WOMAC Pain and Disability Scales-The 5-item WOMAC Pain scale ranges from 0 (no function-related pain) to 20 (severe function-related pain) and asks respondents to rate the intensity of their pain experienced during routine daily activities. Intensity is rated on a five-point Likert scale from "none" to "extreme." The WOMAC Disability Scale is a 17 item scale that assesses the extent of a person's difficulty in performing routine daily activities. The scale ranges from 0 (no knee related functional loss) to 68 (maximal knee related functional loss). ${ }^{23}$ The WOMAC has been studied extensively and its scales have 
been shown to be reliable and valid for quantifying the extent of both pain and disability in patients undergoing knee arthroplasty. ${ }^{23-26}$

\section{Procedures}

Ethical approval of the study was obtained from the IRBs of both institutions and all patients read and signed consent forms prior to participation. After patients consented, they completed all baseline questionnaires. Patients in the coping skills condition then received an 8 session pain coping skills training protocol. Patients in the usual care cohort received no additional care for pain coping. Approximately two months following surgery, all patients were asked to complete a packet of post-treatment questionnaires. For patients in the coping skills training condition, post-treatment questionnaires were collected following completion of the coping skills training which occurred, on average, 67 (SD, 18) days following surgery. Post-surgical questionnaires for the usual care cohort were collected at a mean of 59 (SD, 20) days following surgery. These differences were not significant (independent t-test $=1.37, \mathrm{p}=0.18$ ). All patients completed their questionnaires without assistance from the investigative team.

\section{The Treatment}

The coping skills training protocol was based on the extensive research that has already been done by Keefe, Jensen and others on patients with chronic non-surgical painful conditions. $19 ; 20 ; 22 ; 27-31$ Our overall goal was to customize the intervention to account for the unique needs, interests and concerns of patients who are undergoing knee arthroplasty surgery. Along these lines the intervention addressed the typical recovery of physical function following knee arthroplasty, ${ }^{32}$ the common concerns of patients during the recovery period $^{33}$ and strategies for coping with pain following knee arthroplasty. The intervention was delivered by psychologists with experience and training in using pain coping skills training.

The intervention was designed to be provided in 8 sessions; 1 in-person session approximately 1 month prior to surgery, 6 weekly telephone-based sessions with 3 prior to surgery and three during the 3 weeks following surgery and one in-person session approximately 1 month following surgery. All patients received 8 sessions prior to the two month follow-up. A schedule of 8 sessions over an approximately 2 month period has been shown to be effective in several coping skills trials. ${ }^{31 ; 34-39}$ Use of telephone-based sessions is a relatively new development in behavioral research and is a cost-effective and practical approach for patients, like those following knee arthroplasty who have difficulty with mobility and travel. ${ }^{40 ; 41}$.

To introduce the training, a simplified version of Melzack and Wall's gate control model of pain was used to illustrate that the experience of pain is a complex event influenced by thoughts, feelings and behaviors. ${ }^{42}$ Pain coping strategies were described as skills that can be mastered through home practice. The first applied skill is relaxation training, using a protocol and relaxation tape described by Surwit. ${ }^{43}$ This form of relaxation involved concentrating on muscle tension signals and using them as cues to relax. In addition to this form of relaxation exercise, pleasant imagery was another skill taught, with the rationale that is an additional form of relaxation as well as a form of distraction. ${ }^{44}$ Patients practiced using pleasant imagery and changing from one image to another. A couple of 'pure form' distraction techniques also were provided as additional pain coping tools. These techniques involve brief, intensive focus on salient physical or auditory stimuli in the client's immediate environment. ${ }^{44}$ 
Activity-rest cycling and pleasant activity scheduling ${ }^{45-47}$ were used to reduce pain and to enable patients to pace and increase their activity level. In activity-rest cycling, patients identify activities in which they overexert themselves (e.g., housework or shopping), learn to break those up into periods of activity and rest (e.g., 45 minutes of housework followed by 10 minutes of rest), and gradually increase their activity level as they decrease rest. Patients identified activities they enjoy such as reading, doing crafts and hobbies, or visiting friends and set and recorded weekly activity goals.

Cognitive-restructuring was used to help the patient recognize the relationships between thoughts, feelings and behavior. ${ }^{48}$ These techniques were used to teach patients to identify irrational, maladaptive thoughts and to replace these with alternative, rational coping thoughts. A self-instructional training intervention developed by Turk et al. ${ }^{49}$ was used to help the patient utilize calming self-statements when dealing with severe pain. In addition, basic problem-solving skills involving problem identification, generation of coping alternatives, evaluation of coping alternatives, and selection and implementation of a problem solution were presented and practiced.

In the final session, each patient developed a written maintenance plan that included the list of pain coping skills learned during the study and how they might apply these skills in dealing with future challenges. All content was presented using an investigator developed manual and all patients were given a user friendly patient manual to facilitate training and integration of content.

Our overall goal was to customize the intervention to address the types of catastrophizingrelated issues faced by patients who are undergoing knee arthroplasty surgery. Table 2 lists examples of the types of concerns reported by patients in our study and the types of coping skills used to address the concerns. Concerns varied depending on timeframe relative to surgery and the Table is organized to reflect these varying themes.

A single Psychologist provided coping skills training at each site. The Psychologists at Duke University Medical Center (FJK and DM) completed years of extensive training in the implementation of similar protocols, have used similar protocols in other trials and provides training to other Psychologists participating in trials of pain coping skills training. Prior to using the protocol on patients, the Psychologist at Virginia Commonwealth University Medical Center (WTN) attended a 2 day training program and discussed and practiced each treatment session in the protocol during weekly telephone conferences with FJK and DM using role play and feedback.

\section{Analysis}

Characteristics of the two cohorts were computed and then compared using t-Tests for continuous variables and Pearson Chi square tests for categorical variables. ANCOVA was used to compare the outcomes for the two groups after adjusting for baseline differences. Models were generated for WOMAC Pain and WOMAC Disability measures as well as the PCS scores obtained at baseline and at 2 months following surgery. Cohen's d effect sizes were reported to describe the magnitude of the treatment effect. ${ }^{50}$ SPSS version 17.0.2 was used for all analyses.

\section{RESULTS}

A total of 45 patients from the usual care cohort and 18 patients from the pain coping skills cohort were included in the study (see Table 3 ). Of the 18 patients recruited for pain coping skills training, 15 completed the training. For the three remaining patients, two canceled their surgery and one dropped out of the study after the first training session. 
Patients in the pain coping skills group demonstrated a mean improvement in WOMAC Pain scores of $6.9(\mathrm{sd}=4.7)$ points while patients in the usual care cohort achieved a mean improvement of $2.6(\mathrm{sd}=4.8)$ points (see Table 3$)$. Baseline adjusted discharge score differences among groups for WOMAC Pain were significant at $\mathrm{p}=0.017(\mathrm{~F}=6.02$, $\mathrm{df}=1,60)$. For WOMAC Disability, the pain coping skills cohort improved by $23.5(\mathrm{sd}=$ 12.4) points while the usual care cohort improved by $11.2(\mathrm{sd}=13.9)$ points for a difference among groups of 12.3 WOMAC points. Group differences for baseline adjusted WOMAC Disability discharge scores were significant at $\mathrm{p}=0.023(\mathrm{~F}=5.44, \mathrm{df}=1,60)$. The pain coping skills cohort improved by $19.6(\mathrm{sd}=9.6)$ points on the PCS while the usual care cohort improved by $9.3(\mathrm{sd}=10.0)$ points, a difference of $10.3 \mathrm{PCS}$ points among groups. The baseline adjusted difference in discharge PCS scores was significant at $\mathrm{p}=0.003(\mathrm{~F}=9.96$, $\mathrm{df}=1,59)$. Baseline, follow-up and change scores for the two groups of patients, along with Cohen's d effect sizes appear in Table 3.

\section{DISCUSSION}

We found that improvement in self reported function and function-related pain following pain coping skills training was substantial relative to the usual care group. The mean improvements in WOMAC Disability scores were 14.3 points greater for the pain coping skills group relative to the usual care group. For the WOMAC Pain scale, the mean difference between groups was 4.2 points. These mean differences are substantially larger than the minimal clinically important difference of approximately $10 \%$ of the scale (7 points for WOMAC Disability and 2 or 3 points for WOMAC Pain) reported for WOMAC scores. 51-53 These findings suggest that the pain coping skills intervention holds strong promise for future clinical application. However, our quasi-experimental design precludes firm conclusions regarding the efficacy of the intervention. A definitive trial is clearly warranted based on these preliminary findings.

Pain catastrophizing scale scores demonstrated similar changes to WOMAC scores. Patients in the pain coping skills group had mean change scores that were 10.3 points higher than the usual care group. It is possible that the more dramatic improvements in pain and functional status in the pain coping skills group was mediated by the greater improvements in pain catastrophizing, but our sample size was not large enough to examine this issue. Smeets and colleagues found that pain catastrophizing mediated changes in disability and pain in a randomized trial comparing a cognitive behavioral and a physical treatment to a waiting list group of patients with low back pain. ${ }^{54}$ We suspect that a similar mechanism may mediate effects found in our study; a more definitive study with a larger sample size is needed to test this hypothesis. Clinical trials could also include measures of process variables hypothesized to mediate the effects of catastrophizing on pain, such as measures of neurophysiogiocal responses, cognitive and behavioral coping efforts, ${ }^{12 ; 13}$ and responses of significant others to pain and then determine the extent to which any treatment-related associations between changes in catastrophizing and pain are mediated by these variables. This type of process research tells not only if treatments, such as Pain Coping Skills training, are effective, but can help us to understand why these treatments work. 55

Evidence from randomized clinical trials suggest that pain coping skills training holds promise particularly for patients with chronic musculoskeletal pain. For example, Carson and colleagues found that pain coping skills training resulted in significant improvements over usual care and arthritis education in joint pain ratings and coping efficacy in patients with rheumatoid arthritis. ${ }^{56}$ Smeets and colleagues found that a cognitive behavioral treatment emphasizing pain coping was as effective as a combined treatment of exercise and pain coping and more effective than a wait listed control group at reducing pain and disability. ${ }^{54}$ There is strong evidence from multiple systematic reviews that pain coping 
skills training and other forms of cognitive behavioral therapy are effective in treating patients with various forms of chronic pain. ${ }^{57-59}$ Patients with chronic pain often develop maladaptive thought patterns (i.e., catastrophizing) and behaviors (i.e., guarding or inactivity, perhaps due to fear of movement) that contribute to physical and emotional suffering. The primary goal of these interventions is to aid the patient in reconceptualizing his or her view of pain and role in the process of healing so as to promote increased activity and engagement in usual activities (i.e., pain self-management) rather than passive avoidance.

\title{
Limitations
}

Quasi-experimental designs are viable alternatives to randomized clinical trials particularly when innovative interventions are developed and preliminary testing of the intervention is appropriate prior to making a large financial investment in a more definitive trial. As Campbell and Stanley note, the non-equivalent control group design, as was used in this study, must not be confused with the randomized trial in which patient are randomly assigned to different interventions. ${ }^{60}$ Because patients in our study were not assigned randomly to the two treatment conditions, we cannot be certain that the differences noted were due to the intervention or to pre-treatment differences among the groups. For example, as noted in Table 1, none of the baseline characteristics were significantly different among the two groups. However, the pain coping group had consistently higher WOMAC Pain, Disability and Pain Catastrophizing Scale scores and there were some differences in marital status and race/ethnicity between the treatment groups. While these differences were not statistically significant, they may have had an influence on the outcome, particularly given the small sample sizes in the study. Another internal validity threat to quasi-experimental designs is history. Our usual care group was treated from one to four years prior to the pain coping skills cohort. While surgical and implant procedures may have varied over the study period, we are unaware of evidence indicating that differences we found may have been due to differences in surgical technique over this relatively short time interval.

\section{Conclusions}

Despite the limitations in the design of our study, we found what appears to be very promising effects of pain coping skills training for knee arthroplasty patients with elevated pain catastrophizing. Mean differences in WOMAC Pain, WOMAC Function and Pain Catastrophizing Scale scores between the treatment groups far exceeded clinically important differences. The findings indicate that a definitive trial of pain coping skills training for the subset of knee arthroplasty patients with elevated pain catastrophizing is warranted, particularly because current practice guidelines do not acknowledge the potential effects of catastrophizing in these patients. Despite the preliminary nature of the findings, the results provide an empirical basis for the potential importance of assessing and addressing pain catastrophizing prior to and following surgery.

\section{Acknowledgments}

Grant and Financial Support: The study was partially funded by a grant from the National Institute of Arthritis, Musculoskeletal and Skin Diseases (1R34AR056727-01).

\author{
Abbreviations \\ PCS Pain Catastrophizing Scale \\ WOMAC Western Ontario and McMaster Universities Arthritis Index
}




\section{Reference List}

1. NIH Consensus Panel. NIH Consensus Statement on total knee replacement December 8-10, 2003. J Bone Joint Surg Am. 2004; 86-A(6):1328-1335. [PubMed: 15173310]

2. Noble PC, Conditt MA, Cook KF, Mathis KB. The John Insall Award: Patient expectations affect satisfaction with total knee arthroplasty. Clin Orthop Relat Res. 2006; 452:35-43. [PubMed: 16967035]

3. Brander VA, Stulberg SD, Adams AD, Harden RN, Bruehl S, Stanos SP, Houle T. Predicting total knee replacement pain: a prospective, observational study. Clin Orthop Relat Res. 2003; (416):2736. [PubMed: 14646737]

4. Hawker G, Wright J, Coyte P, Paul J, Dittus R, Croxford R, Katz B, Bombardier C, Heck D, Freund D. Health-related quality of life after knee replacement. J Bone Joint Surg Am. 1998; 80(2):163173. [PubMed: 9486722]

5. Murray DW, Frost SJ. Pain in the assessment of total knee replacement. J Bone Joint Surg Br. 1998; 80(3):426-431. [PubMed: 9619930]

6. Ethgen O, Bruyere O, Richy F, Dardennes C, Reginster JY. Health-related quality of life in total hip and total knee arthroplasty. A qualitative and systematic review of the literature. J Bone Joint Surg Am. 2004; 86-A(5):963-974. [PubMed: 15118039]

7. Edwards RR, Haythornthwaite JA, Smith MT, Klick B, Katz JN. Catastrophizing and depressive symptoms as prospective predictors of outcomes following total knee replacement. Pain Res Manag. 2009; 14(4):307-311. [PubMed: 19714271]

8. Forsythe ME, Dunbar MJ, Hennigar AW, Sullivan MJ, Gross M. Prospective relation between catastrophizing and residual pain following knee arthroplasty: two-year follow-up. Pain Res Manag. 2008; 13(4):335-341. [PubMed: 18719716]

9. Riddle DL, Wad JB, Jiranek WA, Kong X. Preoperative Pain Catastrophizing Predicts Pain Outcome after Knee Arthroplasty. Clin Orthop Relat Res. 2009

10. Roth ML, Tripp DA, Harrison MH, Sullivan M, Carson P. Demographic and psychosocial predictors of acute perioperative pain for total knee arthroplasty. Pain Res Manag. 2007; 12(3): 185-194. [PubMed: 17717610]

11. Sullivan M, Tanzer M, Stanish W, Fallaha M, Keefe FJ, Simmonds M, Dunbar M. Psychological determinants of problematic outcomes following Total Knee Arthroplasty. Pain. 2009; 143(1-2): 123-129. [PubMed: 19304392]

12. Campbell CM, Quartana PJ, Buenaver LF, Haythornthwaite JA, Edwards RR. Changes in situation-specific pain catastrophizing precede changes in pain report during capsaicin pain: a cross-lagged panel analysis among healthy, pain-free participants 1. J Pain. 2010; 11(9):876-884. [PubMed: 20488760]

13. Jensen MP. A neuropsychological model of pain: research and clinical implications 45. J Pain. 2010; 11(1):2-12. [PubMed: 19595637]

14. Sullivan MJ, Thorn B, Haythornthwaite JA, Keefe F, Martin M, Bradley LA, Lefebvre JC. Theoretical perspectives on the relation between catastrophizing and pain 2. Clin J Pain. 2001; 17(1):52-64. [PubMed: 11289089]

15. Cook, TD.; Campbell, DT. Quasi-experimentation : design \& analysis issues for field settings. Boston: Houghton Mifflin; 1979.

16. Sullivan MJL, Bishop S, Pivik J. The Pain Catastrophizing Scale: Development and Validation. Psych Assessment. 1995; 7(4):524-532.

17. Chibnall JT, Tait RC. Confirmatory factor analysis of the Pain Catastrophizing Scale in African American and Caucasian Workers' Compensation claimants with low back injuries. Pain. 2005; 113(3):369-375. [PubMed: 15661446]

18. Osman A, Barrios FX, Gutierrez PM, Kopper BA, Merrifield T, Grittmann L. The Pain Catastrophizing Scale: further psychometric evaluation with adult samples. J Behav Med. 2000; 23(4):351-365. [PubMed: 10984864]

19. Keefe FJ, Caldwell D, Williams DA, Gil KM, Mitchell D. Pain coping skills training in the management of osteoarthritic knee pain: A comparative study. Behavior Therapy. 1990; 21:49-62. 
20. Keefe FJ, Dunsmore J, Burnett R. Behavioral and cognitive-behavioral approaches to chronic pain: recent advances and future directions. J Consult Clin Psychol. 1992; 60(4):528-536. [PubMed: 1506501]

21. Keefe FJ, Kashikar-Zuck S, Opiteck J, Hage E, Dalrymple L, Blumenthal JA. Pain in arthritis and musculoskeletal disorders: the role of coping skills training and exercise interventions. J Orthop Sports Phys Ther. 1996; 24(4):279-290. [PubMed: 8892142]

22. Keefe FJ, Caldwell DS. Cognitive behavioral control of arthritis pain. Med Clin North Am. 1997; 81(1):277-290. [PubMed: 9012765]

23. McConnell S, Kolopack P, Davis AM. The Western Ontario and McMaster Universities Osteoarthritis Index (WOMAC): a review of its utility and measurement properties. Arthritis Rheum. 2001; 45(5):453-461. [PubMed: 11642645]

24. Bellamy N, Buchanan WW, Goldsmith CH, Campbell J, Stitt LW. Validation study of WOMAC: a health status instrument for measuring clinically important patient relevant outcomes to antirheumatic drug therapy in patients with osteoarthritis of the hip or knee. J Rheumatol. 1988; 15(12):1833-1840. [PubMed: 3068365]

25. Escobar A, Quintana JM, Bilbao A, Arostegui I, Lafuente I, Vidaurreta I. Responsiveness and clinically important differences for the WOMAC and SF-36 after total knee replacement. Osteoarthritis Cartilage. 2007; 15(3):273-280. [PubMed: 17052924]

26. Hawker G, Melfi C, Paul J, Green R, Bombardier C. Comparison of a generic (SF-36) and a disease specific (WOMAC) (Western Ontario and McMaster Universities Osteoarthritis Index) instrument in the measurement of outcomes after knee replacement surgery. J Rheumatol. 1995; 22(6):1193-1196. [PubMed: 7674255]

27. Jensen MP, Turner JA, Romano JM, Karoly P. Coping with chronic pain: a critical review of the literature. Pain. 1991; 47(3):249-283. [PubMed: 1784498]

28. Jensen MP, Turner JA, Romano JM. Changes in beliefs, catastrophizing, and cop are ing associated with improvement in multidisciplinary pain treatment. J Consult Clin Psychol. 2001; 69(4):655-662. [PubMed: 11550731]

29. Keefe FJ, Caldwell DS, Queen K, Gil KM, Martinez S, Crisson JE, Ogden W, Nunley J. Osteoarthritic knee pain: a behavioral analysis. Pain. 1987; 28(3):309-321. [PubMed: 2952935]

30. Keefe FJ, Caldwell D, Williams DA, Gil KM, Mitchell D. Pain coping skills training in the management of osteoarthritic knee pain - II: Follow-up results. Behavior Therapy. 1990; 21:435447.

31. Keefe FJ, Caldwell DS, Baucom D, Salley A, Robinson E, Timmons K, Beaupre P, Weisberg J, Helms M. Spouse-assisted coping skills training in the management of osteoarthritic knee pain. Arthritis Care Res. 1996; 9(4):279-291. [PubMed: 8997917]

32. Lingard EA, Katz JN, Wright EA, Sledge CB. Predicting the outcome of total knee arthroplasty. J Bone Joint Surg Am. 2004; 86-A(10):2179-2186. [PubMed: 15466726]

33. Rastogi R, Davis AM, Chesworth BM. A cross-sectional look at pati concerns ent in the first six weeks following primary total knee arthroplasty. Health Qual Life Outcomes. 2007; 5:48. [PubMed: 17678532]

34. Dalton JA, Keefe FJ, Carlson J, Youngblood R. Tailoring cognitive-behavioral treatment for cancer pain. Pain Manag Nurs. 2004; 5(1):3-18. [PubMed: 14999649]

35. Keefe FJ, Caldwell DS, Baucom D, Salley A, Robinson E, Timmons K, Beaupre P, Weisberg J, Helms M. Spouse-assisted coping skills training in the management of knee pain in osteoarthritis: long-term followup results. Arthritis Care Res. 1999; 12(2):101-111. [PubMed: 10513498]

36. Keefe FJ, Blumenthal J, Baucom D, Affleck G, Waugh R, Caldwell DS, Beaupre P, Kashikar-Zuck S, Wright K, Egert J, Lefebvre J. Effects of spouse-assisted coping skills training and exercise training in patients with osteoarthritic knee pain: a randomized controlled study. Pain. 2004; 110(3):539-549. [PubMed: 15288394]

37. Keefe FJ, Anderson T, Lumley M, Caldwell D, Stainbrook D, McKee D, Waters SJ, Connelly M, Affleck G, Pope MS, Weiss M, Riordan PA, Uhlin BD. A randomized, controlled trial of emotional disclosure in rheumatoid arthritis: Can clinician assistance enhance the effects? Pain. 2007 
38. Martire LM, Schulz R, Keefe FJ, Starz TW, Osial TA Jr, Dew MA, Reynolds CF. Feasibility of a dyadic intervention for management of osteoarthritis: a pilot study with older patients and their spousal caregivers. Aging Ment Health. 2003; 7(1):53-60. [PubMed: 12554315]

39. Turner JA, Jensen MP. Efficacy of cognitive therapy for chronic low back pain. Pain. 1993; 52(2): 169-177. [PubMed: 8455964]

40. Bambauer KZ, Aupont O, Stone PH, Locke SE, Mullan MG, Colagiovanni J, McLaughlin TJ. The effect of a telephone counseling intervention on self-rated health of cardiac patients. Psychosom Med. 2005; 67(4):539-545. [PubMed: 16046365]

41. Pariser D, O'Hanlon A. Effects of telephone intervention on arthritis self-efficacy, depression, pain, and fatigue in older adults with arthritis. J Geriatr Phys Ther. 2005; 28(3):67-73. [PubMed: 16386168]

42. Melzack R. Recent concepts of pain. J Med. 1982; 13(3):147-160. [PubMed: 6184428]

43. Surwit, RS. Progressive Relaxation Training Manual. Duke University Medicial Center; 1979.

44. McCaul KD, Malott JM. Distraction and coping with pain. Psychol Bull. 1984; 95(3):516-533. [PubMed: 6399756]

45. Houpt JL, Keefe FJ, Snipes MT. The Clinical Specialty Unit: the use of the psychiatry inpatient unit to treat chronic pain syndromes. Gen Hosp Psychiatry. 1984; 6(1):65-70. [PubMed: 6693028]

46. Keefe FJ. Behavioral assessment and treatment of chronic pain: current status and future directions. J Consult Clin Psychol. 1982; 50(6):896-911. [PubMed: 7174979]

47. Lewinson, PM. The behavioral study and treatment of depression. In: Hersen, M.; Eisler, RM.; Miller, PM., editors. Progress in Behavior Modification. 1975. p. 19-65.

48. Beck, AT.; Rush, AJ.; Shaw, BF.; Emery, G. Cognitive Therapy and Depression. New York: Guilford Press; 1979.

49. Turk, DC.; Meichenbaum, D.; Genest, M. Pain and Behavioral Medicine: A cognitive-behavior perspective. New York: Guilford; 1983.

50. Livingston EH, Elliot A, Hynan L, Cao J. Effect size estimation: a necessary component of statistical analysis. Arch Surg. 2009; 144(8):706-712. [PubMed: 19687373]

51. Angst F, Aeschlimann A, Stucki G. Smallest detectable and minimal clinically important differences of rehabilitation intervention with their implications for required sample sizes using WOMAC and SF-36 quality of life measurement instruments in patients with osteoarthritis of the lower extremities. Arthritis Rheum. 2001; 45(4):384-391. [PubMed: 11501727]

52. Angst F, Aeschlimann A, Michel BA, Stucki G. Minimal clinically important rehabilitation effects in patients with osteoarthritis of the lower extremities. J Rheumatol. 2002; 29(1):131-138. [PubMed: 11824949]

53. Ehrich EW, Davies GM, Watson DJ, Bolognese JA, Seidenberg BC, Bellamy N. Minimal perceptible clinical improvement with the Western Ontario and McMaster Universities osteoarthritis index questionnaire and global assessments in patients with osteoarthritis. $\mathrm{J}$ Rheumatol. 2000; 27(11):2635-2641. [PubMed: 11093446]

54. Smeets RJ, Vlaeyen JW, Kester AD, Knottnerus JA. Reduction of pain catastrophizing mediates the outcome of both physical and cognitive-behavioral treatment in chronic low back pain. J Pain. 2006; 7(4):261-271. [PubMed: 16618470]

55. Kazdin AE. Understanding how and why psychotherapy leads to change 7. Psychother Res. 2009; 19(4-5):418-428. [PubMed: 19034715]

56. Carson JW, Keefe FJ, Affleck G, Rumble ME, Caldwell DS, Beaupre PM, Kashikar-Zuck S, Sandstrom M, Weisberg JN. A comparison of conventional pain coping skills training and pain coping skills training with a maintenance training component: a daily diary analysis of short- and long-term treatment effects. J Pain. 2006; 7(9):615-625. [PubMed: 16942947]

57. Morley S, Eccleston C, Williams A. Systematic review and meta-analysis of randomized controlled trials of cognitive behaviour therapy and behaviour therapy for chronic pain in adults, excluding headache. Pain. 1999; 80(1-2):1-13. [PubMed: 10204712]

58. McCracken LM, Turk DC. Behavioral and cognitive-behavioral treatment for chronic pain: outcome, predictors of outcome, and treatment process. Spine. 2002; 27(22):2564-2573.

[PubMed: 12435995] 
59. Dixon KE, Keefe FJ, Scipio CD, Perri LM, Abernethy AP. Psychological interventions for arthritis pain management in adults: a meta-analysis. Health Psychol. 2007; 26(3):241-250. [PubMed: 17500610]

60. Campbell, DT.; Stanley, JC. Experimental and Quasi-experimental Designs for Research. Boston: Houghton Mifflin Company; 1963. 


\section{Table 1}

Characteristics of the patient samples

\begin{tabular}{|c|c|c|c|}
\hline & $\begin{array}{l}\text { Pain Coping Skills Cohort } \\
(\mathrm{n}=18)\end{array}$ & Historical Cohort $(n=45)$ & $\begin{array}{c}\text { t-Test or Pearson chi- square } \\
\text { Test (p value) }\end{array}$ \\
\hline Age (y) & $63.8(11.5)$ & $60.8(9.9)$ & $-1.0(0.31)$ \\
\hline \multicolumn{4}{|l|}{ Sex } \\
\hline Male & $6(33 \%)$ & $12(26.7 \%)$ & \\
\hline Female & $12(67 \%)$ & $33(73.3 \%)$ & $0.01(0.93)$ \\
\hline \multicolumn{4}{|l|}{ Marital Status } \\
\hline Married & $7(38.9 \%)$ & $27(60 \%)$ & \\
\hline Not Married & $11(61.1 \%)$ & $18(40 \%)$ & $2.3(0.13)$ \\
\hline \multicolumn{4}{|l|}{ Education } \\
\hline Less than high school & $3(17.6 \%)$ & $6(13.6 \%)$ & \\
\hline High School & $6(35.3 \%)$ & $12(27.3 \%)$ & \\
\hline Some college & $4(23.5 \%)$ & $14(31.8 \%)$ & \\
\hline College degree or higher & $4(23.5 \%)$ & $12(27.3 \%)$ & $0.75(0.86)$ \\
\hline \multicolumn{4}{|l|}{ Race/ethnicity } \\
\hline Black & $9(50.0 \%)$ & $13(30.2 \%)$ & \\
\hline White & $8(44.4 \%)$ & $28(65.1 \%)$ & \\
\hline Native American & $1(5.6 \%)$ & $1(2.3 \%)$ & $2.1(0.35)$ \\
\hline Baseline WOMAC Pain Score & $13.1(3.6)$ & $11.2(3.7)$ & $-1.5(0.14)$ \\
\hline Baseline WOMAC Disability Score & $41.5(11.0)$ & $35.3(12.8)$ & $-1.8(0.07)$ \\
\hline Baseline Pain Catastrophizing Scale Score & $29.3(8.9)$ & $25.8(11.1)$ & $-1.25(0.22)$ \\
\hline
\end{tabular}




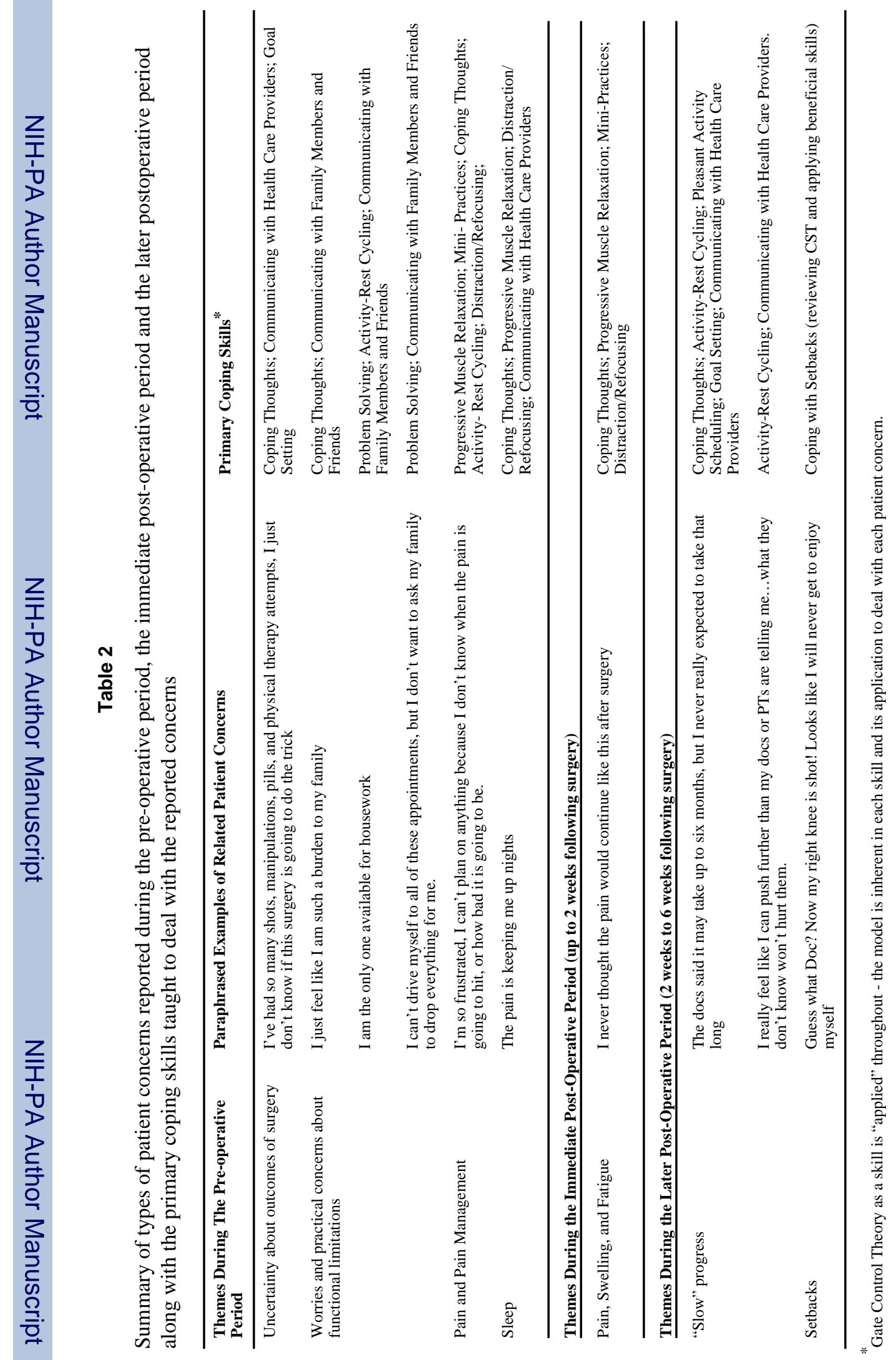




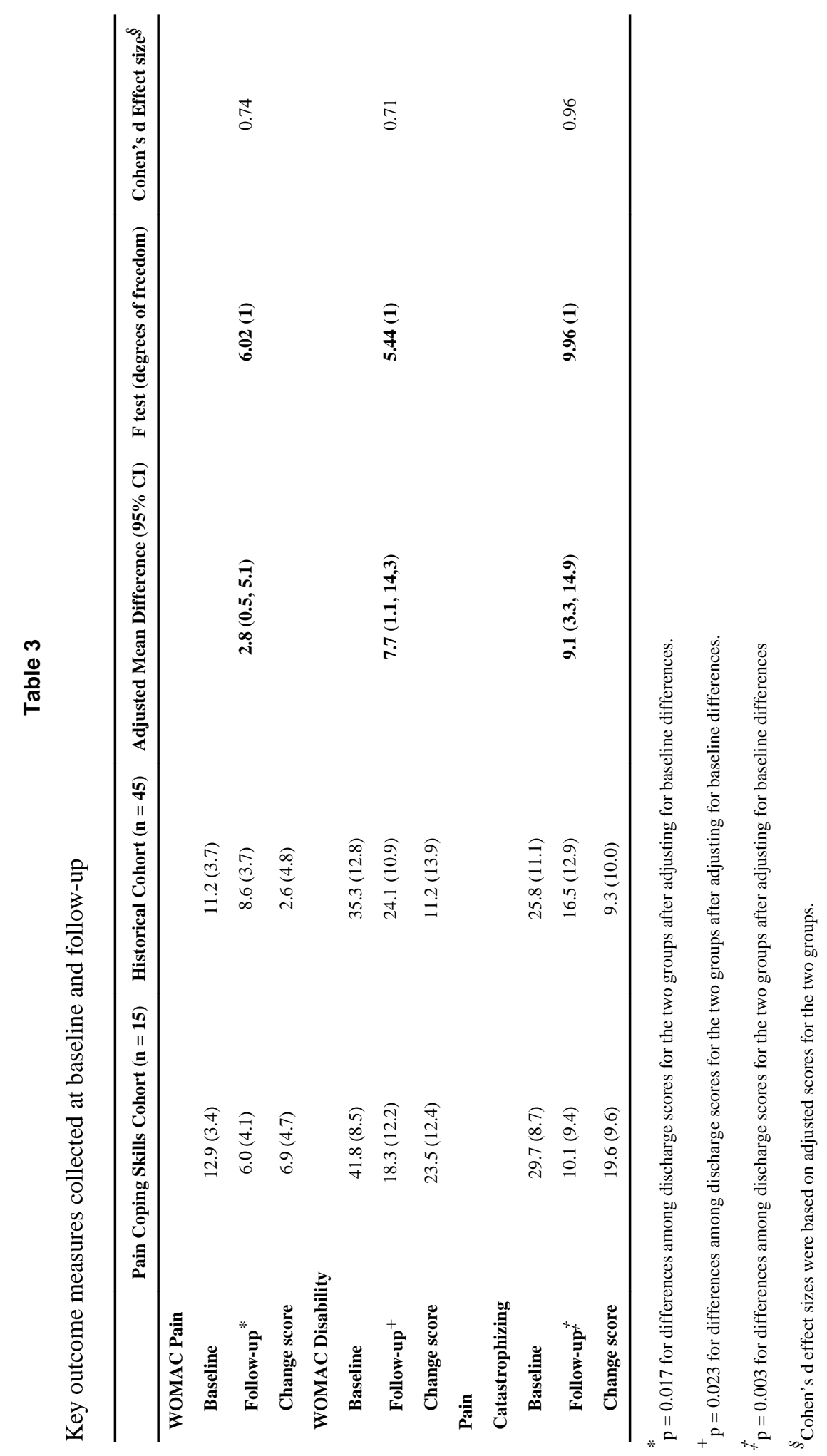

